\title{
Perspectives of incretin mimetics in cardiovascular diseases
}

\author{
João Felipe R. Cardoso, ${ }^{1 *}$ Célia Cohen, ${ }^{2,4}$ Fernanda J. Medeiros,,${ }^{3,4}$ Fabiano M. Serfaty, ${ }^{4}$ Mario F. T. Neves ${ }^{4}$
}

\begin{abstract}
Introduction: Type 2 Diabetes (DM2) is a chronic condition associated with an increased risk of cardiovascular diseases, neuropathies, nephropathies and eye diseases. Incretins (GIP and GLP-1) are hormones important to insulin secretion, and their actions are compromised in DM2 patients. Objectives: This review considers the opportunities and challenges of using incretin mimetics in the treatment of DM2. Methods: Bibliographic review referring to the period from 2000 to 2020, in electronic databases such as Scielo, Lilacs, PubMed, Web of Science. Results: Incretins stimulate insulin secretion by the pancreas in response to nutrient intake, with a lower potential to cause hypoglycemia. In addition, they have a cardioprotective role, reducing blood pressure, improving endothelial and myocardial function, and their use has been associated with a reduction in the risk of cardiovascular events, including cardiovascular mortality. Clinical trials with GLP-1R agonists (GLP-1RA) reduced albuminuria, increased natriuresis, and decreased oxidative stress. In addition, treatment with incretin mimetics reduced the occurrence of the main cardiovascular outcomes related to atherosclerosis, promoted weight loss and improved lipid profile. Conclusion: Studies show the important role of incretin mimetics in the pathophysiology and treatment of DM2, with significant effects in the cardiovascular system. However, its use must be evaluated in relation to its safety and to in which individuals the benefits outweigh the risks associated with the treatment. Thus, its clinical relevance depends on studies with long-term follow-up of patients, with analysis of its impact on mortality and on the development of micro and macrovascular complications.
\end{abstract}

Keywords: Incretins, Diabetes type 2, GLP-1, GIP, DPP-4.

\section{Introduction}

Type 2 diabetes (DM2) is a chronic metabolic disorder characterized by persistent hyperglycemia due to the deficiency of insulin production or action. $\mathrm{DM} 2$ is related to the increased risk of cardiovascular diseases, neuropathies, nephropathies, pancreatitis and cancer. ${ }^{1}$ In Brazil, it is estimated that 13 million people are affected by DM2, and the prevalence increased between 2006 to 2017, around 54\% in men and 28\% in women. Of these, less than $50 \%$ are controlled and have glycated hemoglobin (HbA1c) below 7\%.2,3 Currently, Brazil occupies the 4 th position in the world ranking of people affected by this condition. ${ }^{2}$

The initial phase of the disease is marked by insulin
1. Departamento de Morfologia, Escola de Medicina, Fundação Técnico-Educacional Souza Marques. Rio de Janeiro, RJ, Brazil.

2. Departamento de Nutrição e Dietética, Escola de Nutrição Emília de Jesus Ferreiro. Universidade Federal Fluminense. Niterói, RJ, Brazil

3. Departamento de Nutrição Aplicada, Escola de Nutrição. Universidade Federal do Estado do Rio de Janeiro. Rio de Janeiro, RJ, Brazil.

4. Departamento de Clínica Médica, Universidade do Estado do Rio de Janeiro. Rio de Janeiro, RJ, Brazil.

* Correspondence address:

Fundação Técnico Educacional Souza Marques,

Departamento de Morfologia

Rio de Janeiro, RJ, Brazil.

CEP: $21310-310$

E-mail: E-mail: jfrcardoso@gmail.com

ORCID: https://orcid.org/0000-0001-6391-5986

BJHBS, Rio de Janeiro, 2021;20(1):55-62

DOI: $10.12957 /$ bjhbs.2021.59746

Received on $07 / 07 / 2020$. Approved on 08/31/2020.

resistance and high levels of plasma insulin. In the late stages of the disease, there is an insufficiency in insulin secretion due to $\beta$ cell dysfunction caused, mainly, by deficiency or resistance to incretins. ${ }^{4}$

The main goal of diabetes treatment includes reducing blood glucose without the risk of hypoglycemia, avoiding weight gain, and preventing chronic complications. The selection of the antidiabetic drug is based on the patient's general condition, the presence of comorbidities, fasting and postprandial blood glucose values, HbA1c values and the interaction with other drugs, ${ }^{1}$ as shown in Table 1 .

Conventional therapy may not be enough to reduce $\mathrm{Hb} 1 \mathrm{Ac}$, and it might show adverse effects such as hypoglycemia, gastrointestinal discomfort, heart failure and bone fractures. ${ }^{5}$

This review aims to briefly describe the actions of incretin hormones in the healthy organism and in the pathophysiology of DM2, as well as the different actions of their analogous drugs, such as GLP-1R agonists (GLP-1RA) in the disease control, analyze the effects of the antidiabetic drugs on the risk of adverse effects and on the consequences of DM2 progression. 


\section{Review article}

Table 1. Laboratory criteria for DM2 diagnosis adopted by the SBD

\begin{tabular}{|c|c|c|c|c|}
\hline & Fasting glucose & $\begin{array}{l}\text { Glucose } 2 \mathrm{~h} \text { after } 75 \mathrm{~g} \\
\text { glucose overload }\end{array}$ & Random glucose & $\mathrm{Hb} 1 \mathrm{c}$ \\
\hline Normoglycemia & $<100(\mathrm{mg} / \mathrm{dL})$ & $<140(\mathrm{mg} / \mathrm{dL})$ & - & $<5.7 \%$ \\
\hline $\begin{array}{l}\text { Prediabetes or increased } \\
\text { risk for DM }\end{array}$ & $>100$ e $<126(\mathrm{mg} / \mathrm{dL})$ & $\geq 140$ e $<200(\mathrm{mg} / \mathrm{dL})$ & - & $\geq 5,7$ e $<6.5 \%$ \\
\hline Established diabetes & $\geq 126(\mathrm{mg} / \mathrm{dL})$ & $\geq 200(\mathrm{mg} / \mathrm{dL})$ & $\begin{array}{l}\quad \geq 200 \text { (mg/dL) } \\
\text { Hyperglycemia symptoms }\end{array}$ & $\geq 6.5 \%$ \\
\hline
\end{tabular}

Authorship: Adapted from Guidelines of Brazilian Society of Diabetes 2019-2020.

\section{Incretins}

Incretins belong to the glucagon peptide superfamily, and are mainly represented by the intestinal hormones, glucose-dependent insulinotropic polypeptide (GIP) and glucagon-like peptide (GLP-1). ${ }^{4} \mathrm{GIP}$ is a $42 \mathrm{AA}$ peptide, originated from its precursor pro-GIP, secreted by $\mathrm{K}$ cells located in the duodenum and proximal part of the jejunum. GLP-1 is a peptide with 30 to $31 \mathrm{AA}$, product of the pro-glucagon gene and produced by $\mathrm{L}$ cells, located mainly in the ileum and colon. ${ }^{6,7}$

The secretion of GIP and GLP-1 occurs independently and involve the nervous and endocrine systems. The main stimulus to its secretion is glucose ingestion, followed by lipids and amino acids. ${ }^{6}$ Incretins are responsible for $70 \%$ of insulin secretion during the post-prandial period, being essential in the regulation of glucose homeostasis in people with normal glucose tolerance. ${ }^{8} \mathrm{GIP}$ is responsible for the initial insulin secretion, promoting the release of previously synthesized and stored insulin, whereas GLP- 1 is responsible for the late phase secretion and insulin biosynthesis. ${ }^{4}$

The incretin degradation is catalyzed mainly by the enzyme dipeptidyl peptidase-4 (DPP-4) located in plasma, kidneys, intestinal mucosa, hepatocytes and endothelial cells. ${ }^{5} \mathrm{DPP}-4$ is responsible for maintaining the plasma concentration of GLP-1. ${ }^{5}$ Thus, incretins have a short half-life, with a rapid reduction of its insulinotropic effects ${ }^{6,9}$

The effects of GIP and GLP-1 occur through their specific receptors (GIPR and GLP-1R), from G protein-coupled receptor family. When activated, they promote an increase in cyclic adenosine monophosphate (CAMP) and the exchange protein directly activated by cAMP-2 (EPAC2) in cell10, with phosphorylation of the sulfanylurea 1 receptor (SUR1) subunit, facilitating the closure of the KATP ion channel, which facilitates the membrane depolarization and the volt- age-dependent calcium channel (VCDD) opening, and the mobilization of intracellular calcium stores. ${ }^{9,10}$ The increased intracellular calcium concentration activates insulin secretion, and the increase in intracellular cAMP promotes proinsulin gene transcription. ${ }^{9,10}$

The incretin receptors are found mainly in the $\beta$ cells of the pancreas but are also present in other tissues. GLP-1R is also found in the lungs, kidneys, central nervous system (CNS), enteric and peripherical nervous system, lymphocytes, blood vessels, and heart; while GIPR is found in gastrointestinal tract, adipose tissue, heart, pituitary gland, adrenal cortex, and CNS. 6,11

GLP-1 is primarily responsible for insulin release stimulated by the presence of food in the gastrointestinal tract, known as the "incretin effect", described when glucose administered orally increases in 60\% the insulin secretion compared to intravenous glucose administration. The incretin effect occurs due to the physiological capacity of GLP-1 to stimulate insulin and inhibit glucagon secretion, in a glucose-dependent manner and only during hyperglycemia. ${ }^{1,4}$

The plasma half-life of GLP-1 is about 2 min due to the rapid degradation by the DPP- 4 enzyme. ${ }^{6}$ However, its physiological effects help the glycemic control during fasting and post prandial periods. ${ }^{1} \mathrm{DM} 2$ patients show reduced levels of GLP-1, and therefore, the increase of this hormone to pharmacological levels can correct the hyperglycemia in DM2 patients, which motivated the formulation of strategies for its therapeutic use.,14

\section{Biologic actions of incretins}

\section{Pancreatic Function and Blood Glucose}

The GLP-1RA, beyond insulin secretion, can stimulate the transcription of the pro-insulin gene and insulin biosynthesis, restore biphasic insulin secretion, increase the expression of glucose transporters 
(GLUT-2) and the cytosolic levels of glucokinases in the pancreas $\beta$-cells, promoting a good glycemic control, reduced post-prandial glycemic levels and HbA1c levels. ${ }^{4}$ In addition, they inhibit glucagon secretion in $\alpha$-cells by stimulating the release of inhibitory factors from $\beta$-cells and somatostatin by $\delta$-cells. ${ }^{12}$

The drugs GLP-1RA, when used as a monotherapy or in combination with metformin, reduce $\mathrm{HbA1c} \mathrm{lev-}$ els by 0.7 to $1.1 \%$ in DM2 patients with baseline values close to $8 \%$ and, when associated with insulin, the HbA1c levels is reduced by approximately $5 \%{ }^{7}$ A study with 39 patients with $\mathrm{HbA1c}$ close to $8.5 \%$ showed that Liraglutide, a GLP-1 agonist, promoted a reduction in HbA1c up to $1.5 \%$, with increased biphasic insulin secretion, at a dose of $1.25 \mathrm{mg}$ / day for 14 weeks. ${ }^{13}$

DPP-4 inhibitory drugs (iDPP-4), when used as monotherapy or in combination with metformin, promote a reduction in postprandial blood glucose and HbA1c levels by 0.5 to $1 \%$ in patients with DM2.?

As observed by Mudaliar \& Henry, ${ }_{1}^{14}$ the incretin hormones and their agonists have several effects in addition to glycemic control. They may stimulate cell proliferation and inhibit $\beta$-cell apoptosis, and these effects show clinical correlation with improvement in cell dysfunction and loss of pancreatic parenchyma, commonly seen in DM2 progression.

The activation of GIPR and GLP-1R stimulates the proliferation, neogenesis and survival of $\beta$ cells $^{4,10}$ through the activation of epidermal growth factors. ${ }^{6}$ Studies with rodents with and without diabetes showed that GLP-1R activation promote signal pathways responsible for the growth and differentiation of mature and progenitor $\beta$-cells, like adenosine cyclic monophosphate/protein kinase A/cAMP responsive element binding protein (AMPc/PKA/CREB), phosphatidylinositol 3-kinase (PI3K), and kinases regulated by extracellular signal 1/2 (ERK1/2). ${ }^{15}$

The GIP anti-apoptotic effects on $\beta$-cells are related to cAMP activation and protein kinase B signaling pathways (Akt/PKB). ${ }^{9}$ Studies with INS-1 cells have shown that GIPR activation promotes phosphorylation of the nuclear transcription factor Foxo1, up-regulation of pro-apoptotic BAX genes and down-regulation of BCL-2 genes, ${ }^{16}$ as well as suppressing the translocation of pro-apoptotic proteins Bad and BimEL and Capase-3 activation. ${ }^{17}$

Apart from insulin secretion and glycemic control, incretin mimetics as GLP-1RA are related to cardioprotection, neuroprotection, reduced appetite and decreased gastric motility. ${ }^{4}$

\section{Cardiovascular actions}

Adverse cardiovascular events represent the main cause of mortality and morbidity in diabetic patients.1118 Studies with GLP-1RA suggest many vascular actions such as blood pressure-lowering, improvement of endothelial and myocardial function, and vasodilatation. ${ }^{14}$ In this sense, a recent meta-analysis observed that treatment with GLP-1RA in DM2 patients promoted a $12 \%$ reduction in major adverse cardiovascular events, with a risk ratio of 0.88 for deaths due to cardiovascular causes and 0.84 for non-fatal or fatal stroke. GLP-1RA treatment also reduced mortality from all causes by $12 \%$ and hospital admission for heart failure by $9 \% .{ }^{19}$

In a systematic review and meta-analysis, prolonged therapy with incretin mimetics, iDPP-4 and GLP-1RA, had a lower risk of developing acute myocardial infarction compared with sulfonylurea-based therapies. ${ }^{20}$ Similarly, patients with cardiovascular disease showed improvement in left ventricular ejection fraction, myocardial contraction, and increased myocardial oxygen uptake after treatment with GLP-1.? Gerstein et al. (2019) observed that Dulaglutide had an effect in reducing deaths from cardiovascular events, non-fatal acute myocardial infarction and non-fatal stroke. ${ }^{21}$ The cardioprotective effects are related to the activation of GLP-1R and insulin receptors in the heart, with increased glucose uptake, increased glycogen synthesis and reduced beta-oxidation of fatty acids by the myocardium and increased glucose metabolism. ${ }^{11}$

Experimental studies with cardiomyocytes treated with $10 \mathrm{nM}$ of GLP-1 increased cAMP cellular levels, Akt signaling pathway activation, and increased expression of GLUT1 in sarcolema. ${ }^{11}$ The Akt signaling pathway increases glucose uptake by promoting GLUT1 transporter expression in cell membrane, as it reduces oxidative stress markers, such as glutathione disulfate (GSSG) and thioredoxin reductase; as well, Akt pathway decrease cell apoptosis by up-regulating Bcl-2 gene family.22

Despite clinical studies with DPP-4 inhibitors did not show improvement in cardiovascular outcomes; ${ }^{23}$ experimental in vivostudies with iDDP-4 demonstrated improvement in heart dysfunction and advanced cardiomyopathies, as well as myocardial recovery after ischemia and reperfusion injury, with a decrease in the ischemic area.

However, clinical studies with GLP-1RA observed a significant reduction in major adverse cardiovascular events, considered as possible preventive therapy and 


\section{Review article}

as initial medication after the use of metformin. ${ }^{24} \mathrm{Clin}$ ical studies with GLP-1RA have shown a reduction in blood pressure, vasodilatation, improvement of systolic and diastolic pressures, reduction of norepinephrine levels and a slight increase in heart rate. ${ }^{11,25}$

GLP-1R are also found in the coronary endothelial cell. GLP-1RA therapy, such as Liraglutide, improves endothelial function in DM2 patients through activation of the nitric oxide synthase (eNOS)/NO pathway and anti-inflammatory action on endothelial cells..$^{18,26}$ In vitrostudies with endothelial cells observed increase in nitric oxide production, through the Akt, PKA and AMP-activated protein kinase (AMPK) pathways. ${ }^{18}$

Clinical studies to assess cardiovascular outcomes

The LEADER study followed 9340 patients with DM2 in 32 countries for a minimum of 42 months. The results demonstrated that Liraglutide was able to reduce the risk of cardiovascular death, non-fatal acute myocardial infarction, and non-fatal stroke by $13 \%$ compared to placebo, with an absolute reduction of $1.9 \%$ in risk. Based on this study, the Federal Drugs Administration (FDA) approved the use of Liraglutide to reduce the risk of acute myocardial infarction, stroke, and cardiovascular death in adults with DM2 and pre-established cardiovascular disease. ${ }^{24}$

The SUSTAIN-6 study evaluated Semaglutide, a subcutaneous weekly GLP-1RA that demonstrated a $26 \%$ reduction in the risk of cardiovascular death, non-fatal acute myocardial infarction, and non-fatal stroke for two years, when compared to placebo. ${ }^{25}$

The REWIND study was a multicenter, randomized, double-blind, placebo-controlled study designed to evaluate $1.5 \mathrm{mg}$ of Dulaglutide, a subcutaneous weekly GLP-1RA. This study evaluated a total of 9901 patients from 24 countries, with an average diabetes duration of 10 years, and an average glycated hemoglobin (HbA1c) of 7.3\%. The use of Dulaglutide in the REWIND study reduced cardiovascular adverse events in adults with DM2 with and without a history of cardiovascular disease, with a significant reduction in the composite outcome of cardiovascular death, acute myocardial infarction or stroke. ${ }^{21}$

The patients included in the LEADER ${ }^{24}$ and SUSTAIN- $6^{25}$ studies had similar characteristics, since about $80 \%$ of them had pre-established cardiovascular disease. The REWIND ${ }^{26}$ study, in turn, was different from those carried out previously, because only $31 \%$ of the evaluated patients had a previous diagnosis of cardiovascular disease. In addition, the research had an average follow-up period of more than five years, the longest for a clinical trial on the cardiovascular outcome in the GLP-1RA class.

Clinical trials with other diabetes drugs such as Sitagliptin (TECOS), Alogliptin (EXAMINE), Saxagliptin (SAVOR), Lixisenatide (ELIXA) and Exenatide (EXSCEL), showed zero cardiovascular effects.

\section{Renal actions}

Diabetic Nephropathy has a complex and multifactorial pathophysiology caused by microvascular and hemodynamic events, characterized by progressive decline in renal function, with increased albuminuria, reduced glomerular filtration rate, hypertension and increased morbidity and mortality due to cardiovascular complications. Hypertension impairs glomerular structure and function, while chronic hyperglycemia promotes injury and inflammation in glomeruli and convulated tubules due to advanced glycation products. $^{27}$

Hyperglycemia, hypertension and dyslipidemia promote hemodynamic changes, able to modulate intracellular signaling pathways, transcription factors, cytokines, chemokines, and growth factors, responsible for development and progression of kidney disease, so that 20 to $40 \%$ of DM2 patients end up developing kidney disease. ${ }^{28}$

Clinical studies with GLP-1RA in patients at cardiovascular risk have shown positive effects on renal function, reducing microalbuminuria, glomerulosclerosis, slowing down the glomerular filtration rate decline, increasing natriuresis, reducing oxidative stress, inflammation, and renal fibrosis in patients with DM2 and chronic kidney disease. ${ }^{28,29}$ The reduction in $17 \%$ of renal outcomes occurred mainly due to the reduction in urinary albumin excretion. ${ }^{19}$

In a randomized clinical trial with overweight DM2 patients with preserved renal function, treated with GLP-1RA (Lixisenatide, 20ug) for eight weeks, showed an increase in urinary sodium, chloride and potassium excretion, an increase in urinary $\mathrm{pH}$ and a reduction in urinary magnesium, calcium and phosphate excretion, without changing the glomerular filtration rate, renal flow, glomerular pressure; and without interfering in atrial natriuretic peptide or B-type natriuretic peptide concentrations..$^{30}$

The GLP-1 and its agonists increase diuresis and natriuresis through direct activation of renal tubular cells, promoting phosphorylation and decreasing the 
activity of sodium-hydrogen exchanger 3 (NHE3) in the brush edges of the proximal tubules. ${ }^{29}$ A clinical trial with DM2 patients with persistent albuminuria, treated with Liraglutide for 12 weeks, demonstrated a reduction in activity and plasma concentration of renin and angiotensin II. . $^{31}$

DM2 patients treated with Exenatide and Liraglutide, in randomized clinical trials, benefited from weight loss, reduced systolic pressure, reduced levels of transforming growth factor-b (TGF-b) and collagen type IV, in addition to reduction of albuminuria without a consistent reduction in glomerular filtration. ${ }^{31}$ The effect on renal albumin excretion in clinical studies is corroborated by experimental studies with KK/Ta-Akita mice treated with Liraglutide, presenting a reduction in albuminuria, in mesangial expansion, in superoxide and nicotinamide adenine dinucleotide phosphate (NADPH) production; as well as increased renal activity of cAMP-PKA pathway, usually inhibited in kidney disease. ${ }^{32}$

In addition, GLP1-RA demonstrate antioxidant effects as reported with Exenatide, that suppressed TGF-b concentration, an important factor in diabetic nephropathy pathogenesis. Moreover, its use increased activity of antioxidant agents such as Nuclear factor erythroid 2-related factor 2 (NRF-2), glutathione S-transferase 1P (GST-1P) and NAD(P)H dehydrogenase [quinone] 1 (NQO-1).[33]

\section{Hypolipidemic actions}

The hypothalamus, especially the arcuate nucleus, is responsible for receiving and integrating neural signals and appetite regulation, through two neuronal populations: (1) the appetite inhibiting neurons - pro-opiomelanocortin / cocaine-and amphetamine-regulated transcript (POMC/CART), and (2) the appetite stimulating neurons - neuropeptide $\mathrm{Y} /$ agouti-related protein (NPY/AgRP). ${ }^{34}$

GLP-1R are widely distributed in the central nervous system, ${ }^{14}$ predominantly, in arcuate nucleus and paraventricular nuclei of hypothalamus, and it is suggested that GLP-1 has an important role on the neurological control of gastric emptying, food intake and regulation of glycose homeostasis through insulin secretion and glucose production. ${ }^{26}$ It is suggested that GLP-1RA suppress appetite through ventromedial hypothalamus and solitary tract nucleus by induction of melanocortin and the melanocortin receptor 4 (MCR-4). ${ }^{26}$ However, Baggio \& Drucker ${ }^{35}$ stated that Liraglutide acts indirectly by GABAergic neurons, through inhibition of orexigenic activity of NPY/AgRP neurons and directly on POMC/CART neurons. These findings are corroborated by a study with patients with type 1 diabetes, where Liraglutide promoted the suppression of the plasma concentration of ghrelin after an overnight fasting. ${ }^{26}$

Treatment with GLP-1R agonists promotes weight loss and reduces triacylglycerol (TAG), Apolipoprotein B-48 (ApoB-48), cholesterol and free fatty acids levels. ${ }^{1}$ Experimental studies with rodents receiving a highfat diet and treated with GLP-1RA showed increased expression of acetyl CoA oxidase and acetyl CoA dehydrogenase, suggesting an increase in beta-oxidation of mitochondrial and peroxisomal fatty acids. ${ }^{36}$

Similarly, in a study with 3731 non-diabetic patients with body mass index $>30 \mathrm{~kg} / \mathrm{m}^{2}$, medicated with Liraglutide, and stimulated to make lifestyle modifications, show a reduction in the initial weight by 5 to $10 \%$, and improvement of their metabolic control..$^{37}$ Through pharmacological integrated systems models, the predictions showed that doses of 5 to 10 ug of Exenatide, twice a day, flattened the glucose curve in the morning and at dusk (48 to $49 \%$ ), related to GLP-1RA administration, whereas at noon this effect is discreet (5 to 30\%) due to the low concentration of the agent. This finding demonstrate that postprandial glucose values are affected by delayed effects of gastric emptying of Exenatide. ${ }^{38}$ It is important to highlight that slowing gastric emptying and reduced postprandial glucose levels is only observed in GLP1-RA drugs, such as Exenatide and Liraglutide, not being observed in DPP-4 inhibitors. ${ }^{39}$

In this sense, the weight loss related to GLP-1RA may contribute to cardioprotective effects, since weight loss is related to adiponectins increase in plasma, known for their cardioprotective action, reduction of acute myocardial infarction risk and cardiac hypertrophy ${ }^{40}$

\section{Loss of incretins effects in DM2 patients}

The effects of incretins are reduced in patients with DM2, prediabetics, patients with glucose intolerance, obesity, and insulin resistance with normal glucose tolerance. However, the response of $\beta$-cells to glucose stimulus remains preserved in the initial phase of insulin secretion, while the effects are reduced in the late phase of insulin secretion, but the loss of hormonal efficiency of GIP and GLP- 1 occur distinctly in the progression of DM2. ${ }^{8}$

The intestinal hormone GIP keeps its secretion 
preserved in the progression of the disease. ${ }^{4}$ However, it is believed that the loss of its activity is related to the lower efficiency of GIP receptor. ${ }^{6}$ The proposed mechanisms suggest: receptor mutation; downregulation or desensitization in response to hyperglycemia; decreased intracellular signaling activation; or pancreatic $\beta$ cells disfunction. ${ }^{4}$

On the other hand, there is a reduction in GLP-1 secretion in postprandial period ${ }^{1}$ with the maintenance of GLP-1R activity in DM2 patients; ${ }^{4}$ a fact that contribute to the reduction of insulin secretion and glucagon suppression. ${ }^{1}$

Due the maintenance of GIP insulinotropic activity and decrease in receptor activity, GIP treatment is discouraged in DM2. However, GLP-1 becomes a good therapeutic target in DM2, based in postprandial secretion reduction and the maintenance of the receptor activity in the disease progression. ${ }^{9}$

\section{Incretin mimetics in type 2 diabetes therapy}

The first step in DM2 treatment is the change in diet and lifestyle. However, when these changes are not sufficient, it is necessary to introduce medications to reduce blood glucose and HbA1c levels to values below $7 \%{ }^{2}$

Although the first drug of choice is usually metformin, association with other antidiabetic drugs such as sulfonylureas, thiazolidinediones, meglitinides, alpha-glucosidase inhibitors, GLP-1RA, iDPP-4 or sodium-glucose transport protein 2 (SGLT2) inhibitors can be used when the initial drug of choice alone was not sufficient; and finally, insulin may be necessary in combination or alone. ${ }^{2,5}$

Antidiabetic drugs that increase insulin secretion in a glucose-dependent manner and decrease glucagon secretion is a major advance in DM2 therapy, as these drugs promote important glycemic control without risk of hypoglycemia, added to neurological control of appetite and body weight. ${ }^{14}$

GLP-1RA and iDPP-4 are recommended as a second line in DM2 treatment, usually in combination with metformin. ${ }^{1,2}$ Metformin is a biguanide and its hypoglycemic action occurs through the reduction of hepatic gluconeogenesis and intestinal glucose absorption, increased insulin sensitivity in skeletal muscles1 and stimulating the secretion of GLP- $1 .^{6}$ The association with incretin mimetics, especially iDPP-4, show synergy in hypoglycemic action, as both act in different hypoglycemic mechanisms, thus avoiding clinical hypoglycemia and allowing greater weight loss. ${ }^{4,26}$

Sulfonylureas, a second line of agents in the treatment of DM2, stimulate insulin secretion independently of glucose. The association with incretin mimetics, increase their insulinotropic effects, but this association should be used with caution to avoid severe hypoglycemia. ${ }^{9}$

According to the guidelines of the Brazilian Diabetes Society,,$^{1,2}$ incretin mimetics are indicated for patients with moderate DM2, with fasting blood glucose between 200 to $300 \mathrm{mg} / \mathrm{dL}$, associated with lifestyle changes and metformin.

The incretin mimetics are classified as: (1) GLP-1 receptor agonist drugs with action similar to GLP-1 and degradation resistant, short-lived as Liraglutide and Exenatide, or long-term such as Semaglutide, Lixisenatide, and Dulaglutide; and (2) drugs that inhibit the DPP-4 enzyme, which prevent the hormonal degradation of incretins, increasing their bioavailability and half-life, such as Sitagliptin, Vildagliptin, Saxagliptin, Linagliptin and Alogliptin. ${ }^{1,26}$

\section{Analogs to GLP-1 (GLP-1RA)}

Analogs to GLP-1 are synthetic compounds with homology to the natural GLP-1 hormone and has an agonist action to its specific receptor. They are resistant to the inactivating action of enzyme DPP-4, due to the replacement of the 2 nd AA, usually by glycine, with varying half-life from $2.4 \mathrm{~h}$ to 7 days depending on the drug; but they are still susceptible to renal excretion. 5,27

GLP-1RA have advantages when compared to DDP inhibitors, such as greater stimulus to insulin secretion, a longer half-life than endogenous incretins, greater resistance to DPP-4, delayed gastric emptying, greater reduction in blood glucose, increased satiety, and greater weight loss, ${ }^{4,14}$ However, they have the inconvenience of subcutaneous administration and high incidence of gastric manifestations such as nausea and vomiting, especially Exenatide. ${ }^{4}$

\section{DPP-4 inhibitors (iDPP-4)}

These drugs work by inhibiting the action of the enzyme responsible for the degradation of GIP and GLP-1, and, in this way, increase endogenous hormones bioavailability and half-life, and consequently, the increment of serum levels by up to 4 times, thus promoting inhibition of glucagon secretion, and insulinotropic, anti-hyperglycemic activity. 1,2,26

The advantages over GLP-1 analogue drugs are oral administration, safety and better tolerance, ${ }^{5}$ but its use 
has been linked to an increased risk of infections and there is a need for dose reduction in kidney disease., ${ }^{1,4}$

\section{Conclusion}

Type 2 diabetes is a chronic condition implicated in the development of micro and macrovascular complications. Studies show the important role of incretins in the pathophysiology and treatment of this condition. Much has been learned about the physiology and pharmacology of hormones and drugs based on

\section{References}

1. Sociedade Brasileira de Diabetes. Diretrizes da Sociedade Brasileira de Diabetes 2019-2020. Costa e Forti A, Pires AC, Pittito B de A, Gerchman F, Oliveira JEP de, Zajdenverg L, et al., editors. Editora Clannad; 2019.491 p.

2. Sociedade Brasileira de Diabetes. Conduta Terapeutica no Diabetes Tipo 2: Algoritimo SBD. SBD. 2019;1-40.

3. Nichols GA, Kimes TM, Harp JB, et al. Glycemic Response and Attainment of A1C Goals Following Newly Initiated Insulin Therapy for Type 2 Diabetes. Diabetes Care. 2012;35:495-7.

4. Cernea S. The Role of Incretin Therapy at Different Stages of Diabetes. Rev Diabet Stud. 2011;8(3):323-38.

5. Tasyurek HM, Altunbas HA, Balci MK, et al. Incretins : Their physiology and application in the treatment of diabetes mellitus. Diabetes Metab Res Rev. 2014;30:354-71.

6. Al-sabah S. Molecular Pharmacology of the Incretin. Med Princ Pract. 2016;25(suppl 1):15-21.

7. White WB, Baker WL. Cardiovascular Effects of Incretin-Based Therapies. Annu Rev Med. 2016;67:245-69.

8. Mari A, Bagger JI, Ferrannini E, et al. Mechanisms of the Incretin Effect in Subjects with Normal Glucose Tolerance and Patients with Type 2 Diabetes. PLoS One. 2013;8(9):e73154.

9. Seino Y, Fukushima M, Yabe D. GIP and GLP-1, the two incretin hormones: Similarities and differences. J Diabetes Investig. 2010;1(1):8-23.

10. Chon S, Gautier J. An Update on the Effect of Incretin-Based Therapies on $\beta$-Cell Function and Mass. Diabetes Metab J. 2016;40:99-114.

11. Ussher JR, Drucker DJ. Cardiovascular Biology of the Incretin System. Endocrinol Rev. 2012;33(2):187-215

12. Campbell JE, Drucker DJ. Pharmacology , Physiology, and Mechanisms of Incretin Hormone Action. Cell Metab [Internet]. 2013;17:819-37

13. Vilsbøll T, Brock B, Perrild $H$, et al. Liraglutide, a oncedaily human GLP-1 analogue , improves pancreatic B-cell function and arginine-stimulated insulin secretion during hyperglycaemia in patients with Type 2 diabetes mellitus. Diabet Med. 2008;25:152-6.

14. Mudaliar S, Henry RR. The incretin hormones: from scientific discovery to practical therapeutics. Diabetologia. 2012;55:1865-8.

15. Klinger S, Poussin C, Debril M, et al. Increasing GLP1 - Induced $\mathrm{N}_{\mathrm{L}}$-Cell Proliferation by Silencing the Negative Regulators of Signaling cAMP Response Element Modulator-a. Diabetes. 2008;57:584-93.

16. Trümper A, Trümper K, Hörsch D. Mechanisms of mitogenic and anti-apoptotic signaling by glucose-dependent incretins. These agents show significant effects in the control of HbA1c, blood glucose and body weight, as well as, on several organs and systems, which allows a broader performance. However, the use of incretin mimetics should be evaluated based on their safety and when the benefits outweigh the risks associated with treatment. The clinical relevance will depend on long-term follow-up studies, with analysis of its impact on mortality and in the development of micro and macrovascular complications.

insulinotropic polypeptide in $\beta$ (INS-1) -cells. J Endocrinol. 2002;174:233-46.

17. Ehses JANA, Casilla VR, Doty TIM, et al. Glucose-Dependent Insulinotropic Polypeptide Promotes $\beta$-(INS-1) Cell Survival via Cyclic Adenosine Monophosphate-Mediated Caspase-3 Inhibition and Regulation of p38 Mitogen-Activated Protein Kinase. Endocrinology. 2003;144(10):4433-45.

18. Higashi $Y$. Incretin-related drugs and cardiovascular events: $A$ comparison of GLP-1 analogue and DPP-4 inhibitor. J Cardiol. 2017;69:508-10.

19. Kristensen SL, Rorth R, Jhund OS, et al. Cardiovascular, mortality, and kidney outcomes with GLP-1 receptor agonists in patients with type 2 diabetes: a systematic review and meta-analysis of cardiovascular outcome trials. The Lancet, 2019;7(10):776-785.

20. Chou C, Chang Y, Yang J, et al. Effect of Long-term IncretinBased Therapies on Ischemic Heart Diseases in Patients with Type 2 Diabetes Mellitus: A Network Meta- analysis. Sci Rep [Internet]. 2017;7(1):15795.

21. Gerstein HC, Colhoun HM, Dagenais GR, et al. Dulaglutide and cardiovascular outcomes in type 2 diabetes (REWIND): a double-blind, randomised placebo-controlled trial. Lancet. 2019;394:121-30.

22. Zhao $\mathrm{Y}, \mathrm{Hu} X$, Liu Y, et al. ROS signaling under metabolic stress : cross-talk between AMPK and AKT pathway. Mol Cancer. 2017;16:1-12.

23. Wu S, Cipriani A, Yang Z, et al. The cardiovascular effect of incretin-based therapies among type 2 diabetes: a systematic review and network meta-analysis. Expert Opinion on Drug Safety, 2018;17(3):243-249.

24. Marso SP, Daniels GH, Brown-Frandsen K, et al. Liraglutide and cardiovascular outcomes in type 2 diabetes. N Engl J Med. 2016;375(4): 311-22.

25. Marso SP, Bain SC, Consoli A, et al. Semaglutide and cardiovascular outcomes in patients with type 2 diabetes. $\mathrm{N}$ Engl J Med. 2016;375: 1834-44.

26. Dandona P, Ghanim H, Chaudhuri A. Incretins: Beyond type 2 diabetes. Diabetes, Obes Metab. 2018;20(Suppl 1):59-67.

27. Baar MJB Van, Aart AB Van Der, Hoogenberg K, et al The incretin pathway as a therapeutic target in diabetic kidney disease: a clinical focus on GLP-1 receptor agonists. Ther Adv Endocrinol Metab Rev. 2019;10:1-11.

28. Fioretto P, Frascati A. Role of incretin based therapies in the treatment of diabetic kidney disease. Diabetes Mellit [Internet] 2018;21(5):395-8.

29. Macisaac RJ, Thomas MC. Effects of Diabetes Medications Targeting the Incretin System on the Kidney. Clin J Am Soc Nephrol 13. 2018;13:321-3. 


\section{Review article}

30. Tonneijck L, Smits MM, Muskiet MHA, et al. Acute renal effects of the GLP-1 receptor agonist exenatide in overweight type 2 diabetes patients: a randomised. Diabetologia [Internet]. 2016;59:1412-21.

31. Scholten BJ Von, Persson F, et al. The effect of liraglutide on renal function: A randomized clinical trial. Diabetes. Obes Metab Metab. 2017;19(2):239-47.

32. Fujita $\mathrm{H}$, Morii T, Fujishima $\mathrm{H}$, et al. The protective roles of GLP$1 \mathrm{R}$ signaling in diabetic nephropathy : possible mechanism and therapeutic potential. Kidney Int. 2013;85:579-89.

33. Ghanim H, Chaudhuri A, Abuaysheh S, et al. Exenatide Suppresses TGFB1: A Novel Potential Role for GLP-1 Agonists in Nephropathy. Diabetes. 2016;65(Suppl 1):A131.

34. Perry B, Wang Y. Appetite regulation and weight control: the role of gut hormones. Nutr Diabetes [Internet]. 2012;2:e26-7.

35. Baggio LL, Drucker DJ. Glucagon-like peptide-1 receptors in the brain: controlling food intake and body weight. J Clin Invest. 2014;124(10):4223-6.

36. Mells JE, Fu PP, Sharma S, et al. Glp-1 analog, liraglutide, ameliorates hepatic steatosis and cardiac hypertrophy in C57BL/6J mice fed a Western diet. Am J Physiol Gastrointest Liver Physiol. 2012;302:G225-35.

37. Pi-Sunyer X, Astrup A, Fujioka K, et al. new england journal. N Engl J Med. 2015;373(1):11-22.

38. Voronova V, Zhudenkov K, Penland RC, et al. Exenatide effects on gastric emptying rate and the glucose rate of appearance in plasma: A quantitative assessment using an integrative systems pharmacology model. Diebetes, Obes Metab. 2018;20:2034-8

39. Suganuma Y, Shimizu T, Sato T, et al. Magnitude of slowing gastric emptying by glucagon-like peptide-1 receptor agonists determines the amelioration of postprandial glucose excursion in Japanese patients with type 2 diabetes. J Diabetes Investig. 2020;11(2):389-399.

40. Shibata R, Sato K, Pimentel DR, et al. Adiponectin protects against myocardial ischemia- reperfusion injury through AMPK- and COX-2 - dependent mechanisms. Nat Med. 2005;11(10):1096-103. 